\title{
MORIN PREVENTS ULTRAVIOLET-B RADIATION-INDUCED PHOTOCARCINOGENESIS THROUGH ACTIVATING THROMBOSPONDIN-1 IN THE MOUSE SKIN
}

\author{
ANJUGAM C ${ }^{1}$, SRIDEVI ${ }^{2 *}$,RAJENDRA PRASAD N ${ }^{3}$, AGILAN BALUPILLAI ${ }^{3}$
}

${ }^{1}$ Department of Biochemistry, Vinayaka Mission Medical College, Vinayaka Missions Research Foundation (Deemed to be University)., Salem, Tamil Nadu, India. ${ }^{2}$ Department of Biotechnology, Vinayaka Mission Engineering College, Vinayaka Missions Research Foundation (Deemed to be University)., Salem, Tamil Nadu, India. ${ }^{3}$ Department of Biochemistry and Biotechnology, Annamalai University, Annamalainagar, Tamil Nadu, India. Email: drsridevimuruhan@gmail.com

Received: 27 July 2017, Revised and Accepted: 14 November 2017

\section{ABSTRACT}

Objective: In this study, we investigated whether morin, a natural flavonoid, could able to inhibit ultraviolet B (UVB)-induced carcinogenesis in the skin of Swiss albino mice.

Methods: The mice were exposed to UVB radiation $\left(180 \mathrm{~mJ} / \mathrm{cm}^{2}\right)$ on weekly thrice for 30 weeks, and morin was administered intraperitoneal and topical application $1 \mathrm{~h}$ before UVB exposure. UVB radiation induces the overexpression of cyclooxygenase-2 (COX-2), inducible nitric oxide synthase (iNOS), vascular endothelial growth factor (VEGF), transforming growth factor-beta (TGF- $\beta$ ), and Bcl-2 genes.

Results: Morin significantly prevented UVB-induced activation of COX-2, iNOS, VEGF, TGF- $\beta$, and Bcl-2 expression in mouse skin. Thrombospondin-1 (TSP), a novel endogenous factor, inhibits angiogenesis and inflammation.

Conclusion: The present study illustrates that the protective effect of morin against UVB-induced carcinogenesis may be modulated through activation of TSP-1 in UVB-exposed Swiss albino mice.

Keywords: Ultraviolet B radiation, Morin, Photocarcinogenesis, Thrombospondin-1.

(c) 2018 The Authors. Published by Innovare Academic Sciences Pvt Ltd. This is an open access article under the CC BY license (http://creativecommons. org/licenses/by/4. 0/) DOI: http://dx.doi.org/10.22159/ajpcr.2018.v11i2.21652

\section{INTRODUCTION}

The intensity levels of ultraviolet (UV) radiation reaching ground have increased due to the stratospheric ozone depletion which leads to the dramatic increase in the number of skin cancers in the human population. Chronic exposure to UV radiation is the main environmental factor that causes both non-melanoma and melanoma skin cancers and increasing at a high rate for approximately 2-3 million new cases each year worldwide [1]. Epidemiological and clinical studies designate that UV radiation, mainly ultraviolet B (UVB) (290-320 nm) radiation, induces that erythema occurs approximately $4 \mathrm{~h}$ after exposure, peaks around $8-24 \mathrm{~h}$, and it promotes photocarcinogenesis [2]. UVB radiation-induced inflammation and angiogenesis is the next stage of development of neoplasia. Cyclooxygenase-2 (COX-2) and inducible nitric oxide synthase (iNOS) are understood to be involved in such processes since they participate in the synthesis of mediators of inflammation and carcinogenesis [3].

The UVB radiation-induced inflammatory response stimulated skin angiogenesis and carcinogenesis. Vascular endothelial growth factor (VEGF) is a proangiogenic factor which is overexpressed by UVB radiation [4]. Several carcinogen studies have reported that overexpression of VEGF is involved in enhanced skin vascularization with increased numbers of tortuous and hyperpermeable blood vessels, resulting in angiogenesis and carcinogenesis [5]. Numerous mechanisms have been proposed to inhibit UVB radiation-induced skin carcinogenesis. However, protective mechanisms for UVB radiationinduced skin cancer are still unclear. Therefore, endogenous inhibitors are involved to inhibit inflammation, proliferation, and angiogenesis

Thrombospondin-1 (TSP-1), a potent endogenous molecule, is involved in inhibiting angiogenesis. TSP-1 has regulated an extensive role such as tumor cell adhesion, proliferation, apoptosis, inflammation and immune response, tumor invasion, and metastatic dissemination [6,7]. Xin et al., in 2014, reported that TSP-1 could inhibit angiogenesis through VEGF and HIF-1 $\alpha$ [8].
Apoptosis is a major mechanism by which potentially harmful cells are eliminated during UVB radiation exposure [9]. Bcl-2 family proteins play an important role in a programmed cell death pathway. The mechanism of Bcl-2 family proteins to protect apoptosis is the prevention of mitochondrial depolarization [10]. The ratio between antiapoptotic (Bcl-2) and proapoptotic (Bax) has been suggested as a primary event in determining the susceptibility to apoptosis through maintaining the integrity of the mitochondria and inhibiting the activation of the caspase cascade [11].

In the past decades, the beneficial effects of naturally occurring plantderived substances had been explored for prevention and treatment of the UVB radiation-mediated skin disorders [12,13]. A variety of flavonoids and polyphenols from the plant-derived phytochemicals have been reported to show antioxidative, anti-inflammatory, antimicrobial, and anticancer effects [14]. Morin is one of the bioflavonoids, originally isolated from members of the Moraceae family. It is present in different herbs and fruits such as onion, almond, red wine, and osage orange [15]. Morin shows evidence of some pharmacological properties such as antioxidant, anti-inflammatory, and anticancer effects [16-19]. It has been reported that morin decreases the oxidative damage of cardiovascular cells [20] and can inhibit the growth of human leukemia HL-60 cells through cell cycle arrest [21]. So far, there are not many studies evaluating the effect of morin on UVB radiation-induced photocarcinogenesis. In this study, we evaluated the effect of morin on UVB radiation-induced photocarcinogenesis through activating TSP-1 in the mouse skin.

\section{METHODS}

Chemicals

Morin, monoclonal antibodies anti(COX-2), anti(iNOS), anti(VEGF), anti-transforming growth factor-b1 (TGF-b1), antitumor suppressor gene (p53), anti-Bax, anti-Bcl-2, (TSP-1), and anti- $\beta$-actin anti-mouse 
and goat anti-mouse IgG-HRP polyclonal antibody were purchased from Sigma Chemical Co., St. Louis, MO. All other reagents used were of analytical grade obtained from SD Fine Chemical, Mumbai, and Fisher Inorganic and Aromatic Limited, Chennai.

\section{Preparation of morin and mode of administration}

Morin (15 mg/kg.b.wt.) was dissolved in $0.5 \%$ dimethyl sulfoxide (DMSO) for administration; then, morin was prepared by dissolving in $0.5 \%$ DMSO and made up with water to obtain the final required concentration.

\section{Photocarcinogenesis protocol}

Normal healthy male Swiss albino mice aged 6 weeks weighing 18-20 g were used. The mice were randomly divided into 6 different treatment groups with 8 mice in each group: Group 1: Vehicle control, Group 2: Morin-IP (intraperitoneal) alone, Group 3: Morin-Topical alone, Group 4: UVB irradiation $\left(180 \mathrm{~mJ} / \mathrm{cm}^{2}\right)$ control, Group 5: Morin-IP+ UVB, andGroup 6: Morin-Topical + UVB. UVB irradiation performed at $180 \mathrm{~mJ} / \mathrm{cm}^{2}$ thrice a week for 30 weeks. One hour prior UVB exposure, mice were pretreated with administration of morin.

\section{UVB irradiation procedure for experimental animals}

The mice skin was shaved (dorsal portions) with an electric clipper (Oster A2).

At least 2 days before treatment, the hair removing cream was applied for Anne French, Geoffery Manners, and Bombay, India. The experiment used only the mice which showed no signs of hair regrowth. The cream was washed with water.

The dorsal skin was exposed to UVB radiation, Philips TL40W/12 RS lamp (Heber Scientific, Chennai) emitting $320 \mathrm{~nm}$. The lamp is mounted $20 \mathrm{~cm}$ above the table where the mice placed on. As per the standard photocarcinogenesis protocol (Fig. 1), mice were UVB irradiated $\left(180 \mathrm{~mJ} / \mathrm{cm}^{2}\right) 3$ times a week until the end of the protocol. After the experimental period, the mice were sacrificed by decapitation and the dorsal skins were collected for biochemical and molecular analysis. The procedures for mice were UVB irradiated as described earlier [22].

\section{Evaluation of tumor growth}

The UVB-irradiated skin of the mice was examined once a week for tumor appearance. Growths that were $>1 \mathrm{~mm}$ in diameter and that persisted for at least 2 weeks were defined as tumors and recorded. The dimensions of all the tumors on each mouse were recorded at the termination of the experiment. Tumor volumes were calculated using the Hemiellipsoid model formula: Tumor volume $=1 / 2(4 \pi / 3)(1 / 2)$ (w/2) h, where l=length, w=width, and h=height. At the termination of the experiment, mice were sacrificed and skin samples were collected for the analysis of various biomarkers of interest. The incidence and multiplicity of carcinoma were also recorded in the same experiment.

\section{Histopathological analysis of mice tumors}

For histopathological study, three mice from each group were perfused with cold physiological saline, followed by formalin $(10 \%$ formaldehyde). The mice skin tissue was excised immediately and fixed in $10 \%$ formalin, then dehydrated on treatment with a series of different concentration of ethanol, and embedded in paraffin wax. 3-5 $\mu \mathrm{m}$ thick sections were prepared using a microtome and stained with hematoxylin and eosin. The specimens were evaluated with a light microscope. All histopathological changes were examined by pathologist.

Analysis of VEGF, TGFß1, Bax, and Bcl-2 in photocarcinogenic mice skin

Specific antigens present in the mice skin tissue sections were detected by treating with appropriate antibodies. Visualization of the antigens was observed under a light microscope (Olympus BX51, Japan). Immunohistochemical images of VEGF, TGF- $\beta 1$, Bax, and Bcl-2 expression were performed in formalin-fixed and paraffin-embedded

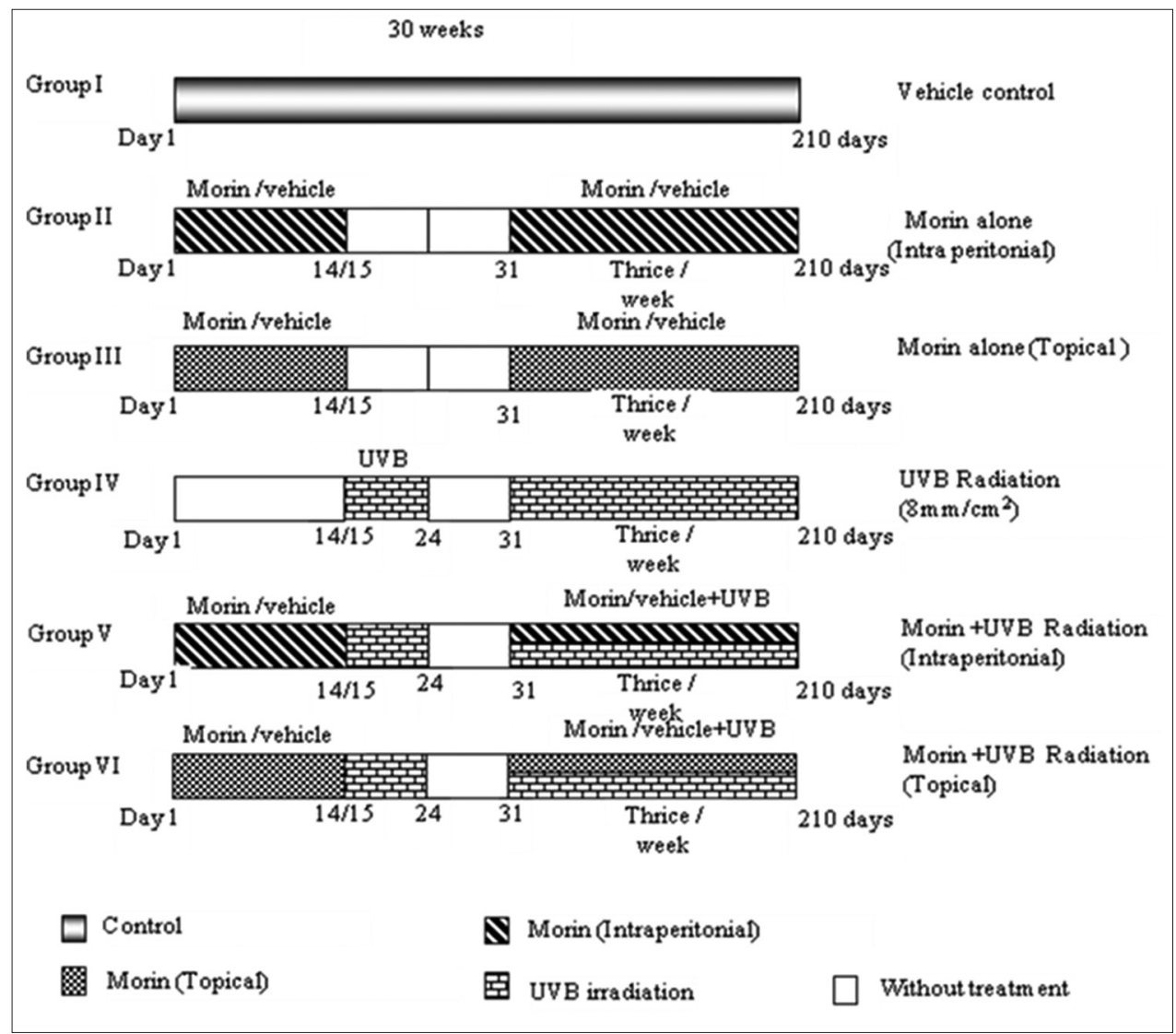

Fig. 1: Photocarcinogenesis protocol 
mice tissue section on a Biogenex system using the standard protocol. The section was pretreated with heat-mediated antigen retrieval with Tris EDTA buffer ( $\mathrm{pH} \mathrm{6)}$ for 20 min. Then, the section was incubated using HRP conjugated compact polymer systems. Diaminobenzidine was used as the chromogen. The section was then counter stained with hematoxylin and mounted with Distyrene Plasticizer Xylene.

\section{Western blot analysis for inflammatory markers expression}

The western blot was carried out by the method of Towbin (1979) [23]. Immunoblot analysis was carried out for COX-2, iNOS, and TSP-1 protein expression in morin plus UVB-irradiated mice skin tissue. The results were normalized to $\beta$-actin gene expression. Following the protein estimation, the samples were separated using sodium dodecyl sulfatepolyacrylamide gel electrophoresis gel electrophoresis and were blotted on polyvinylidene fluoride (PVDF) membrane. After the block, the primary antibody was added and allowed to bind to the protein followed by washing (which removes added, to detect the primary antibody). Then, the PVDF membranes were washed with TBST thrice with 10 mins interval, and the protein expressions were detected by chemiluminescence substrate (LI-COR, USA). The images were acquired by Image Studio software (LI-COR, USA).

\section{Molecular docking}

Molecular docking was performed on Centos 6 Linux workstation using [24]. GLIDE-6.0 searches were performed for understanding docking interactions between morin and TSP-1. All molecular modeling was carried out using OPLSAA (Optimized Potential Liquid Simulation for All Atom) force field [25]. Ligprep 2.3 module (Schrodinger) was employed for morin preparation. The three-dimensional crystal structure of TSP-1 (Protein Data Bank (PDB) Id: 2ES3) was downloaded from the PDB (http://www.rcsb.org). Protein preparation wizard of Schrodinger was used for preparation of TSP-1. No hydrogen atoms were minimized until the average root mean square deviation reached a default value of 0.3 A. Sitemap 2.3 was used to understand binding site in the ligand-binding domain (LBD) of the TSP-1 [24]. Induced fit docking was performed to predict morin binding modes and structural movements in the LBD region of TSP-1 using Glide and Prime modules. The prepared proteins were loaded on the workstation and the Grid values were calculated about $20 \AA$ to cover the entire active site amino acids. About 20 conformational images were created and analyzed for the best conformation pose based on the docking score and glide energy.

\section{Statistical analysis}

All the values were expressed as the mean of six $(n=6)$ determination unless otherwise stated. The data were statistically analyzed using one-
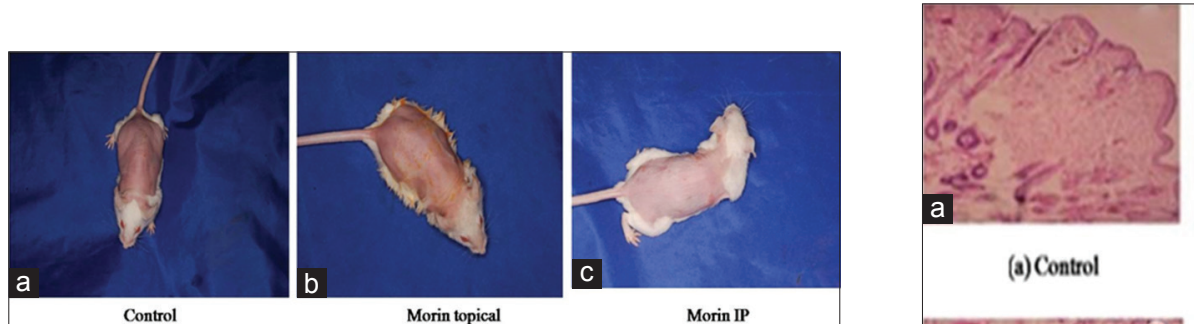

Morin topical

Morin IP
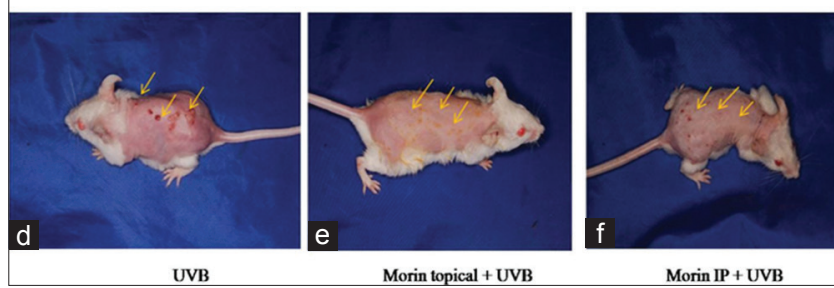

Morin topical + UVB

Morin IP+ UV

Fig. 2: Effect of chronic ultraviolet B (UVB) exposure on skin tumor formation. Morin prevents UVB radiation-induced photocarcinogenesis. Arrow marks indicate tumors growth in UVB-exposed and/or morin-pretreated mice skin. (a) Control. (b) Morin topical. (c) Morin IP. (d) UVB. (e) Morin topical+UVB. (f) Morin IP+UVB way analysis of variance on the Statistical Package for Social Sciences, and the group means were compared to Duncan's multiple range test (DMRT). The results were considered statistically significant if the $\mathrm{p}<0.05$ levels.

\section{RESULTS}

Morin inhibits UVB-induced skin carcinogenesis

Chronic whole-body UVB-irradiated mice developed skin tumor (Fig. 2d). Morin topical and intraperitoneal administration before each UVB-exposure prevented tumor formation in the skin of Swiss albino mice (Fig. 2e and f). When compared with UVB-irradiated mice skin, there was no significant difference in body weight gain on the control and morin control (Fig.2 a-c)

Tumor growth was not observed in the skin of mice which were treated with morin (intraperitoneal and topical). Fig. 3 shows that total of 36 tumors/8 mice and $300 \mathrm{~mm} 3$ tumor volumes were recorded in theUVB irradiated mice. UVB irradiation plus intraperitoneal and topical treatment with Morin ( $2.5 \mathrm{mg} /$ mouse) showed only 12 tumors/8 mice and $80 \mathrm{~mm}^{3}$ tumor volumes (fourth and fifth group of mice)

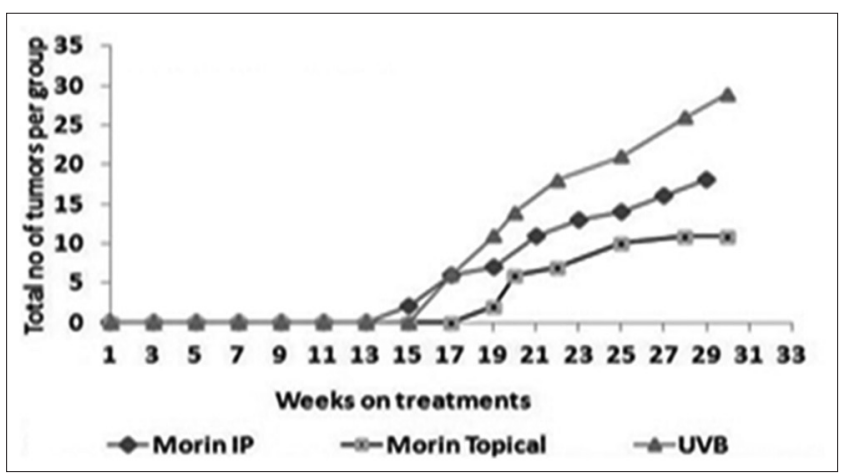

Fig. 3: Effect of morin on ultraviolet B (UVB) induces skin carcinogenesis in Swiss albino mice. Swiss albino mice were UVB-irradiated and/or treated with morin (intraperitoneal and topical) $1 \mathrm{~h}$ before each UVB exposure. Tumor number per mouse $\left(\mathrm{mm}^{3}\right)$ was recorded. Morin pretreatment prevented UVB-induced photocarcinogenesis incidence in experimental animals
(a)Control



(d) UVB

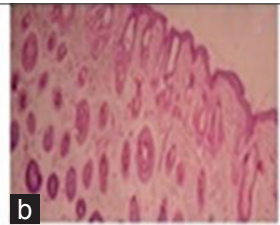

(b) Morin topical

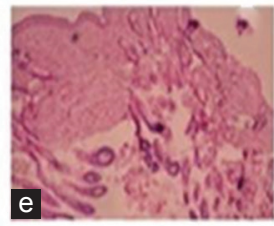

'(e) Morin topical + UVB

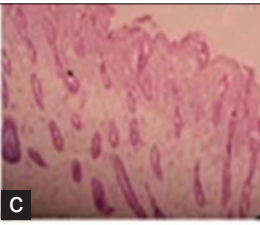

(c) Morin IP

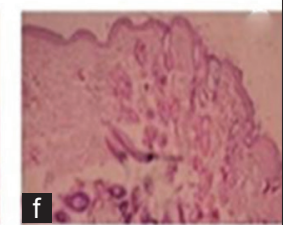

(I) Morin IP+ UVB

Fig. 4: Effect of morin on cancerous lesions in the whole-body ultraviolet B (UVB)-exposed mice skin tumor. Hematoxylin and Eosin stained skin cross-sections showed (i) dysplastic

epithelium and (ii) epithelial island with keratin pearl, hyperccccplasia, dysplastic features, and microinvasive SSC in UVB-exposed animals. (a) Control. (b) Morin topical. (c) Morin IP. (d) UVB. (e) Morin topical+UVB. (f) Morin IP+UVB 
Morin attenuates photocarcinogenesis in the skin of Swiss albino mice

Moreover, control non-irradiated mouse skin and morin alone treated mouse skin were observed no histopathological features (Fig. 4a-c). We examined the effects of morin on UVB-irradiation significantly increased hyperplasia and squamous cell carcinoma (SCC) features in the dermis when compared with the non-UVB-exposed mice skin (Fig. 4d). Conversely, both intraperitoneal and topical administration of morin treatment significantly prevent the UVB-induced hyperplasia and microinvasive SCC and degradation of collagen fibers in the mice skin (Fig.4e and f) when compared with UVB irradiated control mice skin.

Effect of morin on UVB-induced inflammatory responses in Swiss albino mice

COX-2 and iNOS were involved in inflammatory responses. There was a significant overexpression of COX-2 and iNOS observed in UVBirradiated mice skin when compared to non-irradiated mice (Fig. 5). Pre-treatment with morin (intraperitoneal and topical) prior UVB exposure decreased COX-2 and iNOS expression when compared to UVB alone treatment.
Effect of morin on UVB-induced angiogenic and apoptotic markers in swiss albino Mice

Fig. 6 shows that chronic UVB exposure enhanced expression of VEGF, TGF- $\beta 1$, and Bcl2 and decreased Bax expression when compared to the non-UVB-exposed mice skin. Pre-treatment with morin (intraperitoneal and topical) prior UVB exposure decreased VEGF, TGF- $\beta 1$, and Bcl- 2 and enhanced Bax expression in epidermal and dermal cells.

\section{Morin on TSP-1 activation in UVB-exposed Swiss albino mice}

Western blotting analysis showed that there was a downregulation of TSP1 expression in UVB-irradiated mice skin. Treatment with morin before UVB exposure prevented loss of TSP-1 expression, and morin treatment significantly activates the expression of TSP-1 in the mouse skin (Fig. 7a and b). Furthermore, morin was interact with TSP-1 (PDB code: 2ES3) and increases its activity. The glide energy score of morin was found to be $-38.206 \mathrm{kcal} / \mathrm{mol}$ and (Fig. 7c) the docking was found to be $-6.582 \mathrm{kcal}$ mol1. Morin possesses hydrogen bond interaction with TSP-1 at the site of Gln 120, Thr 85, and Glu 90. The amino acid residues such as Gln 96, Gly 96, Gly 184, and Val 186 showed hydrophobic interactions with morin and TSP-1.

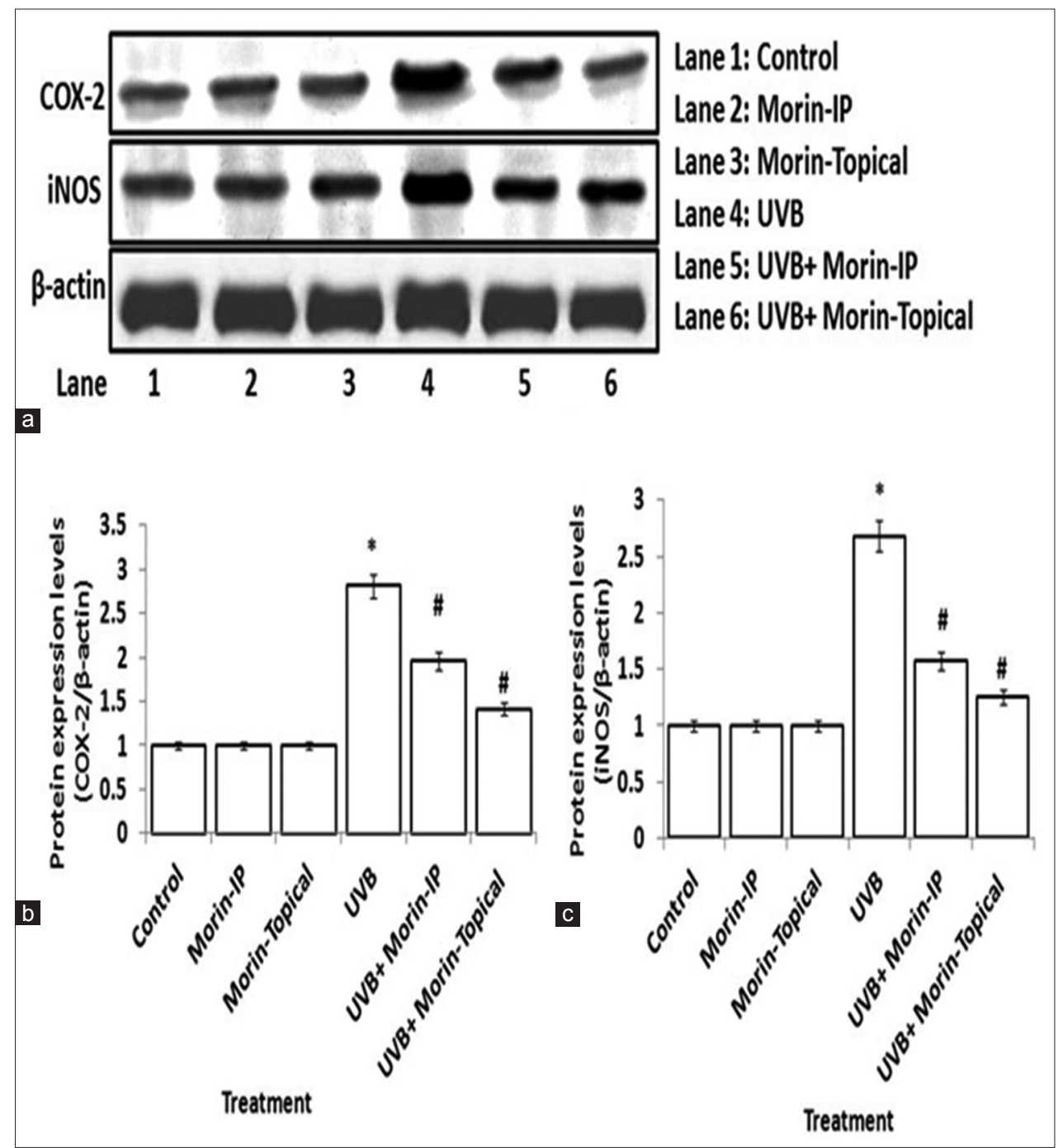

Fig. 5: (a-c) Effects of morin on ultraviolet B (UVB)-induced cyclooxygenase-2 (COX-2) and inducible nitric oxide synthase (iNOS) expression in mouse skin. Dorsal mice skin ( $\mathrm{n}=8$ per treatment group) was treated with morin $1 \mathrm{~h}$ before irradiation with UVB $\left(180 \mathrm{~mJ} / \mathrm{cm}^{2}\right)$. Mice were sacrificed, after the end of treatment period, total tissue lysates were analyzed for COX-2 and iNOS expression by western blot analysis. Quantification of COX-2 and iNOS immunoblot was normalized to $\beta$-actin followed by statistical analysis of relative image density. Values are given as means \pm standard deviation of six experiments in each group. Values not sharing a common superscript differ significantly at $\mathbf{p}<0.05$ (Duncan's multiple range test) 


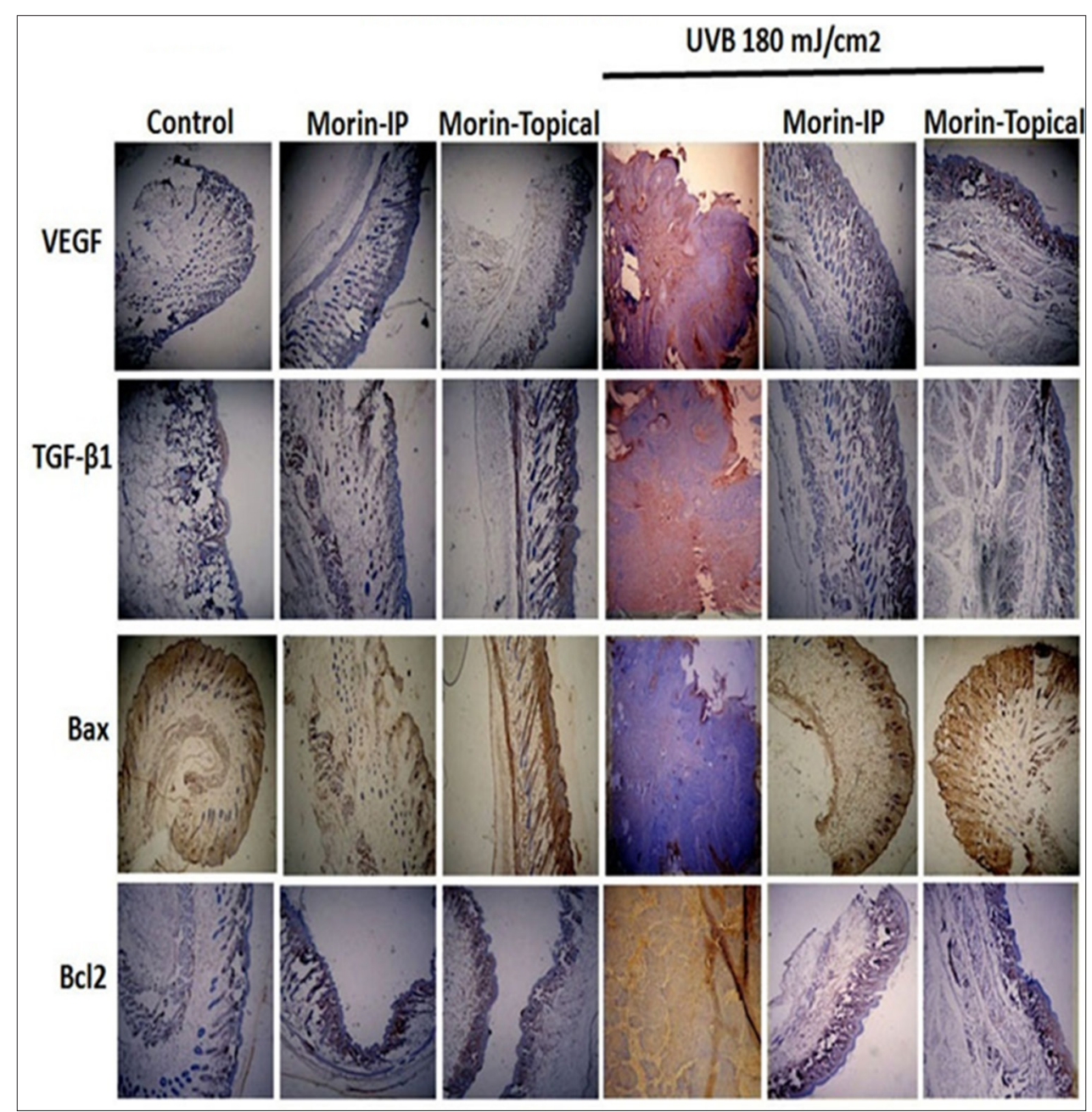

Fig. 6: Inhibitory effects of morin on ultraviolet B (UVB)-induced proliferative markers expressions in mouse skin. Dorsal portion of mice skin ( $\mathrm{n}=\mathbf{8}$ per treatment group) was treated with morin $1 \mathrm{~h}$ before irradiation with UVB $\left(180 \mathrm{~mJ} / \mathrm{cm}^{2}\right)$. Mice were sacrificed, after the end of treatment period, formalin-fixed and paraffin-embedded skin sections from different treatment groups were subjected to immunohistochemical analysis of vascular endothelial growth factor, transforming growth factor-beta $1, \mathrm{Bax}$, and $\mathrm{Bcl}_{2}$ expressions.

Representative photomicrographs $(4 \times)$ showing positive staining appear as brown spots in different treatment groups

\section{DISCUSSION}

UV radiation specifically (UVB 280-320 nm) promotes skin cancer development by mutagenic, immunosuppressive, and oxidative stressinducing mechanisms; however, certain antioxidants may counteract and prevent UVB-induced photodamage [26]. Polyphenols and flavonoids are widely spread in plants, mainly in fruits and vegetables, contributing to their flavor and color [27]. The flavonoids constitute a large group of compounds containing a number of phenolic hydroxyl groups attached to ring structures and it possesses significant antioxidant activity $[28,29]$.

In the present study, we observed significant tumor numbers in 30 week UVB-exposed mice skin (Fig. 3) and histological observation confirmed the presence of SCC, hyperplasia, and dysplastic features in the mice skin (Fig. 4d). Conversely, morin treatment prevented the UVB-induced SCC, hyperplasia, and dysplastic features in the mice skin (Fig. 4e and f). It has been previouly reported that morin incorporated into a topical solution of ascorbic acid and $\alpha$-tocopherol doubled photoprotection to solar-simulated irradiation of skin as measured by both erythema and sunburn cell formation [30]. Histopathological studies confirmed that morin prevented UVB-induced SCC in mice skin.

$\mathrm{UV}$ radiation is considered a major etiologic factor for the development of skin cancers. UVB-mediated inflammation has been correlated with skin cancer development. COX-2 is an inducible enzyme implicated in the synthesis of prostaglandins from arachidonic acid. Overexpression of COX-2 stimulates the production of inflammatory cytokines under UVB exposure, which was linked to the pathophysiology of inflammation and cancer [31]. iNOS is an another key enzyme which is involved in the synthesis of nitric oxide from the amino acid l-arginine. The inhibition of COX-2 and iNOS expression has been shown to suppress the development of skin tumor [32]. Previous report stated that sulindac, a specific inhibitors for COX-1/COX-2 expression that may reduce prostaglandin production leads to the inhibition of UVB-induced skin cancer [33]. Our results also clearly mentioned that administration of morin significantly inhibited UVB-induced overexpression of COX-2 and iNOS in mouse skin.

VEGF is a proangiogenic factor that regulates vascular permeability [34]. The enhancement of vascular permeability implicated in inflammation, angiogenesis, and other pathological processes [35]. The expression of VEGF is also increased by epidermal growth factor (EGF) and TGF- $\alpha$ and TGF- $\beta$ which is involved in the formation tumor genesis [36]. In skin, it is possible that each TGF- $\beta$ isoform could have differential effects during various stages of carcinogenesis. TGF- $\beta 1$ expression appears to be associated with a more differentiated tumor phenotype [37]. The studies have been documented that during UVB overexposure on the skin there is overexpression of VEGF and TGF $\beta$ has been 


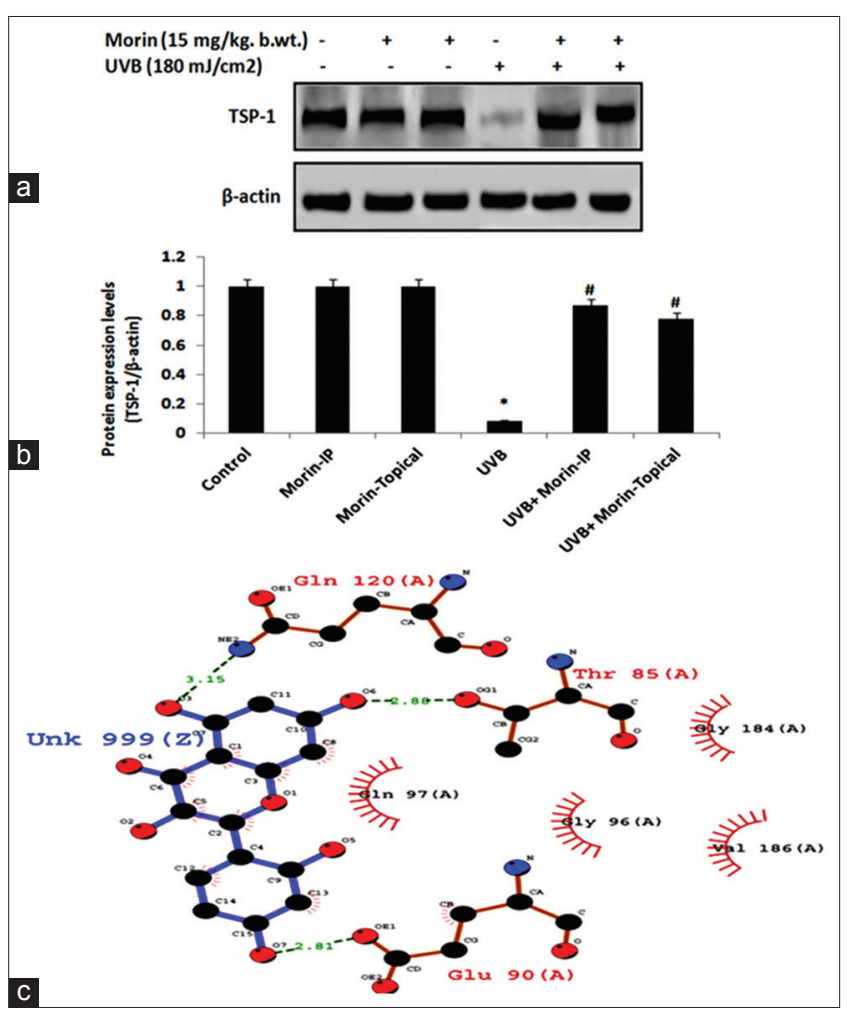

Fig. 7: Morin on ultraviolet B (UVB)-induced activation of thrombospondin-1 (TSP-1) expression in mouse skin. (a) Dorsal mice skin ( $\mathrm{n}=\mathbf{8}$ per treatment group) were treated with morin $1 \mathrm{~h}$ before irradiation with UVB $\left(180 \mathrm{~mJ} / \mathrm{cm}^{2}\right)$. Mice were sacrificed after the end of the treatment period, total tissue lysates were analyzed for TSP-1 expression by western blot analysis. (b) Quantification of TSP-1 immunoblot was normalized to $\beta$-actin followed by statistical analysis of relative image density. Values are given as means \pm standard deviation of six experiments in each group. Values not sharing a common superscript differ significantly at $\mathbf{p}<\mathbf{0 . 0 5}$ (Duncan's multiple range test). (c) Pymol view of the ligand binding domain region of TSP-1 with morin

overexpressed $[38,39]$. In this study, morin treatment decreased the expression of VEGF and TGF- $\beta$ in UVB-exposed mice skin. Previous results demonstrate that resveratrol inhibits expression of TGF- $\beta$ and its isoforms in UVB-irradiated mice skin [40]. Furthermore, VEGF has been inhibited by polyphenols and flavonoid-derived chemicals in UVBirradiated different animal models and in vitro studies $[8,41]$.

UVB radiation-induced inflammation and cell proliferation directly associated with skin angiogenesis result in the development of skin carcinogenesis. TSP-1 inhibits angiogenesis and other carcinogenic events. TSP-1 regulates inflammation by engaging several receptors and by modulating activities of other secreted factors [42]. In this study, morin was found to be an activator of TSP- 1 and subsequently modulates UVB-induced inflammatory and angiogenesis. Similarly, apigenin, a similar flavonoid, activates TSP-1 and thereby inhibits angiogenesis and carcinogenic events in the mouse skin [8]. TSP-1 possesses hydrophobic residue in the ligand binding domain (LBD) region that forms hydrogen bonds (Gln 120, Thr 85, and Glu 90) and hydrophobic interactions (Gln 96, Gly 96, Gly 184, and Val 186) with morin. This binding interaction might be due to the presence of -OH groups in the LBD regions. Ligand exposure also considered to be one of the reasons for strong binding interaction (Fig. 7). Thus, morin behaves as ligand and activates TSP-1, thereby modulating UVB-induced inflammation and angiogenesis in the mouse skin.

In general, UVB exposure could lead to cell death known as apoptosis or programmed cell death. When there is a prolonged exposure of UVB radiation, the cells escape from apoptosis and it goes to carcinogeneic [43]. Bcl-2 family members play crucial role in regulating apoptosis. Overexpression of Bcl-2 has been reported to enhance the survival in various cells and prevent apoptosis induced by a wide range of signals including UV irradiation [44,45]. In this study, chronic exposure of UVB radiation to mice skin escaped the apoptotic process. We observed that morin treatment enhances the expression of Bax and decreased Bcl-2 protein in UVB-irradiated mice. Previously, it has been reported that morin induces apoptosis by induction of BAD and Bcl-2 protein in human leukemic cells [46]. Thus, topical and intraperitoneal administration of morin inhibits UVB radiation-induced oxidative stress, inflammation, angiogenesis, and carcinogenesis probably through activation of TSP-1 in the mouse skin.

\section{AUTHORS CONTRIBUTION}

The objective, experimental part of the work and writing of the manuscript was done by the first author Mrs. Anjugam. Checked the manuscript and references part was done by Dr. Agilan Balupillai. Revision of the manuscript was done by the corresponding authors Dr.Sridevi and Dr.Rajendra Prasad. Authors certifies that this manuscript has not been published anywhere before its appearance in Asian Journal of Pharmaceutical and Clinical Research.

\section{CONFLICT OF INTERESTS}

None.

\section{REFERENCES}

1. WHO. Ultraviolet Radiation and the Intersun Programme Report; 2012. Available from: http://www.who.int/uv/health/en.

2. Anitha T. Medicinal plants used in skin protection. Asian J Pharm Clin Res 2012;5:35-8.

3. Reuter S, Gupta SC, Chaturvedi MM, Aggarwal BB. Oxidative stress, inflammation, and cancer: How are they linked? Free Radic Biol Med 2010;49:1603-16.

4. Trompezinski S, Pernet I, Schmitt D, Viac J. UV radiation and prostaglandin E2 up-regulate vascular endothelial growth factor (VEGF) in cultured human fibroblasts. Inflamm Res 2001;50:422-7.

5. Jung SK, Lee KW, Byun S, Lee EJ, Kim JE, Bode AM, et al. Myricetin inhibits UVB-induced angiogenesis by regulating PI-3 kinase in vivo. Carcinogenesis 2010;31:911-7.

6. Jeanne A, Schneider C, Martiny L, Dedieu S. Original insights on thrombospondin-1-related antireceptor strategies in cancer. Front Pharmacol 2015;6:252.

7. Kazerounian S, Yee KO, Lawler J. Thrombospondins in cancer. Cell Mol Life Sci 2008;65:700-12.

8. Tong X, Mirzoeva S, Veliceasa D, Bridgeman BB, Fitchev P, Cornwell $\mathrm{ML}$, et al. Chemopreventive apigenin controls UVB-induced cutaneous proliferation and angiogenesis through huR and thrombospondin-1. Oncotarget 2014;5:11413-27

9. Lee $\mathrm{CH}, \mathrm{Wu} \mathrm{SB}$, Hong $\mathrm{CH}$, Yu HS, Wei YH. Molecular mechanisms of UV-induced apoptosis and its effects on skin residential cells: The implication in UV-based phototherapy. Int J Mol Sci 2013;14:6414-35.

10. Zamzami N, Marchetti P, Castedo M, Zanin C, Vayssière JL, Petit PX, et al. Reduction in mitochondrial potential constitutes an early irreversible step of programmed lymphocyte death in vivo. J Exp Med 1995;181:1661-72.

11. Jyoti BC, Balaji KS, Jayarama S, Wethroe K. Evaluation of apoptogenic effects of Averrhoa bilambi extract on enrlich ascites carcinoma bearing mice. Asian J Pharm Clin Res 2016;9:348-52.

12. Korać RR, Khambholja KM. Potential of herbs in skin protection from ultraviolet radiation. Pharmacogn Rev 2011;5:164.

13. krishnan KP, Narayanaswamy N. Botanicals as sunscreens: Their role in the prevention of photoaging and skin cancer. Int J Res Cos Sci 2011;1:1-2.

14. Pandey KB, Rizvi SI. Plant polyphenols as dietary antioxidants in human health and disease. Oxid Med Cell Longev 2009;2:270-8.

15. Nandhakumar R, Salini K, Devaraj SN. Morin augments anticarcinogenic and antiproliferative efficacy against 7, 12-dimethylbenz (a)-anthracene induced experimental mammary carcinogenesis. Mol Cell Biochem 2012;364:79-92.

16. Prahalathan P, Kumar S, Raja B. Morin attenuates blood pressure and oxidative stress in deoxycorticosterone acetate-salt hypertensive 
rats: A biochemical and histopathological evaluation. Metabolism 2012;61:1087-99.

17. Merwid-Ląd A, Trocha M, Chlebda E, Sozański T, Magdalan J, Ksiądzyna D, et al. Effects of morin-5'-sulfonic acid sodium salt (NaMSA) on cyclophosphamide-induced changes in oxido-redox state in rat liver and kidney. Hum Exp Toxicol 2012;31:812-9.

18. Fang SH, Hou YC, Chang WC, Hsiu SL, Chao PD, Chiang BL, et al. Morin sulfates/glucuronides exert anti-inflammatory activity on activated macrophages and decreased the incidence of septic shock. Life Sci 2003;74:743-56

19. Lee MH, Cha HJ, Choi EO, Han MH, Kim SO, Kim GY et al. Antioxidant and cytoprotective effects of morin against hydrogen peroxide-induced oxidative stress are associated with the induction of Nrf-2-mediated HO-1 expression in V79-4 Chinese hamster lung fibroblasts. Int J Mol Med. 2017;39:672-680.

20. Kok LD, Wong YP, Wu TW, Chan HC, Kwok TT, Fung KP, et al. Morin hydrate: A potential antioxidant in minimizing the free-radicalsmediated damage to cardiovascular cells by anti-tumor drugs. Life Sci 2000;67:91-9.

21. Kuo HM, Chang LS, Lin YL, Lu HF, Yang JS, Lee JH, et al. Morin inhibits the growth of human leukemia HL-60 cells via cell cycle arrest and induction of apoptosis through mitochondria dependent pathway. Anticancer Res 2007;27:395-405.

22. Vaid M, Katiyar SK. Molecular mechanisms of inhibition of photocarcinogenesis by silymarin, a phytochemical from milk thistle (Silybum marianum L. Gaertn.) (Review). Int J Oncol 2010;36:1053-60.

23. Towbin H, Staehelin T, Gordon J. Electrophoretic transfer of proteins from polyacrylamide gels to nitrocellulose sheets: Procedure and some applications. Proc Natl Acad Sci U S A 1979;76:4350-4.

24. Schrodinger Suite. The Induced Fit Docking Protocol. New York, NY: Schrodinger, LLC; 2009.

25. Induced Fit Docking Protocol. Glide, Version 5.5. New York, NY: Schrodinger, LLC; 2009.

26. Lee EH, Faulhaber D, Hanson KM, Ding W, Peters S, Kodali S, et al. Dietary lutein reduces ultraviolet radiation-induced inflammation and immunosuppression. J Invest Dermatol 2004;122:510-7.

27. Rice-Evans C, Miller N, Paganga G. Antioxidant properties of phenolic compounds. J Invest Dermatol 1997;2:152-9.

28. Dai J, Mumper RJ. Plant phenolics: Extraction, analysis and their antioxidant and anticancer properties. Molecules 2010;15:7313-52.

29. Dzialo M, Mierziak J, Korzun U, Preisner M, Szopa J, Kulma A. The Potential of Plant Phenolics in Prevention and Therapy of Skin Disorders. Int J Mol Sci. 2016;17:160.

30. Lin FH, Lin JY, Gupta RD, Tournas JA, Burch JA, Selim MA, et al. Ferulic acid stabilizes a solution of vitamins $\mathrm{C}$ and $\mathrm{E}$ and doubles its photo protection of skin. J Invest Dermatol 2005;125;826-32.

31. Meeran SM, Punathil T, Katiyar SK. IL-12 deficiency exacerbates inflammatory responses in UV-irradiated skin and skin tumors. J Invest Dermatol 2008;128:2716-27.

32. Cheng W, Li J, You T, Hu C. Anti-inflammatory and immunomodulatory activities of the extracts from the inflorescence of Chrysanthemum indicum Linne. J Ethnopharmacol 2005;101:334-7.

33. Liedtke AJ, Crews BC, Daniel CM, Blobaum AL, Kingsley PJ, Ghebreselasie K, et al. Cyclooxygenase-1-selective inhibitors based on the (E)-2'-des-methyl-sulindac sulfide scaffold. J Med Chem 2012;55:2287-300.

34. Bates DO. Vascular endothelial growth factors and vascular permeability. Cardiovasc Res 2010;87:262-71.

35. Balupillai A, Prasad RN, Ramasamy K, Muthusamy G, Shanmugham M, Govindasamy K, et al. Caffeic acid inhibits UVBinduced inflammation and photocarcinogenesis through activation of peroxisome proliferator-activated receptor- $\gamma$ in mouse skin. Photochem Photobiol 2015;91:1458-68.

36. Hoeben A, Landuyt B, Highley MS, Wildiers H, Van Oosterom AT, De Bruijn EA, et al. Vascular endothelial growth factor and angiogenesis. Pharmacol Rev 2004;56:549-80.

37. Santibanez JF. Transforming growth factor-Beta and urokinasetype plasminogen activator: Dangerous partners in tumorigenesisimplications in skin cancer. ISRN Dermatol 2013;2013:597927.

38. Glick AB. TGF? Back to the future: Revisiting its role as a transforming growth factor. Canc Biol Ther 2004;3:276-83.

39. Yano K, Kadoya K, Kajiya K, Hong YK, Detmar M. Ultraviolet B irradiation of human skin induces an angiogenic switch that is mediated by up regulation of vascular endothelial growth factor and by down regulation of thrombospondin-1. Br J Dermatol 2005;152:115-21.

40. Kim KH, Back JH, Zhu Y, Arbesman J, Athar M, Kopelovich L, et al. Resveratrol targets transforming growth factor- $\beta 2$ signaling to block UV-induced tumor progression. J Invest Dermatol 2011;131:195-202.

41. Ambothi K, Prasad NR, Balupillai A. Ferulic acid inhibits UVBradiation induced photo carcinogenesis through modulating inflammatory and apoptotic signaling in swiss albino mice. Food Chem Toxicol 2015;82:72-8

42. Miyata Y, Sakai H. Thrombospondin-1 in urological cancer: Pathological role, clinical significance, and therapeutic prospects. Int $\mathrm{J}$ Mol Sci 2013;14:12249-72.

43. Reagan-Shaw S, Breur J, Ahmad N. Enhancement of UVB radiationmediated apoptosis by sanguinarine in $\mathrm{HaCaT}$ human immortalized keratinocytes. Mol Canc Ther 2006;5:418-29.

44. Frenzel A, Grespi F, Chmelewskij W, Villunger A. Bcl2 family proteins in carcinogenesis and the treatment of cancer. Apoptosis 2009; 14:584-96.

45. Plati J, Bucur O, Khosravi-Far R. Apoptotic cell signaling in cancer progression and therapy. Integr Biol (Camb) 2011;3:279-96.

46. Park C, Lee WS, Go SI, Nagappan A, Han MH, Hong SH, et al. Morin, a flavonoid from moraceae, induces apoptosis by induction of BAD protein in human leukemic cells. Int J Mol Sci 2014;16:645-59. 